was a time when the patient suffered only from so-called "chronic bronchitis." It is highly important to state this, because the time to treat an incurable disease like emphysema is before structural changes occur-it is the early so-called chronic bronchitis that we must treat. If this is a part of the asthma syndrome then we must concentrate on asthma to get rid of chronic bronchitis and emphysema. It is important to know whether we are dealing with two diseases or one, with asthma and bronchitis, or asthma alone.

The Name.-Certain writers refuse to recognize the existence of a primary chronic bronchitis under any circumstances ("Diseases of the Chest," Norris and Landis, B. 268). I confess to being sceptical, and I would suggest a not altogether original view, but one not generally held in England, that what we call chronic bronchitis in the beginning is not primarily an inflammatory condition at all, it is not primarily a disease of the air passages, it is the part of the asthma syndrome which is always more or less present in cases of asthma, namely the vasosecretory swelling of the bronchial mucous membrane. I will enumerate one or two facts which appear to support this view :-

Chronic bronchitis is inherited, a disease passed on from father to son. This alone would make us suspect it was not originally an inflammation. In "chronic bronchitis" the classical signs of inflammation are themselves absent, there is no rise of temperature. Chronic bronchitis, like asthma, is periodic and fluctuating and lasts a longer period of time than inflammations do.

In many cases of chronic bronchitis the clinical picture is asthma where the bronchitic symptoms, and turgescence of the bronchial mucous membrane, overshadow the muscular spasm of the bronchi. Cases which commence as chronic bronchitis go on tó typical paroxysmal attacks of asthma. The two states have many things in common. Chronic bronchitis sometimes begins in infancy, it frequently recurs year after year, it is periodic, it is progressive, it has the complications and sequelæ of bronchial asthma, and the remedy which in my experience is often effective in asthma is often effective in chronic bronchitis, namely, sodium or potassium iodide.

The same remedy for what is really the same disease. Finally, the microscopic appearance of sections of chronic bronchitis are consistent with a noninflammatory sero-cellular condition.

In conclusion, I am inclined to think that we overlook an important part of the asthma syndrome, namely, the vaso-secretory turgescence of the bronchial mucous membrane and submucous tissue, and that the chronic bronchitis which so often accompanies and complicates asthma is primarily not a chronic bronchitis at all, but the clinical manifestation of the second part of the syndrome (the first part being muscular constriction).

\section{FEVER OF OBSCURE ORIGIN IN CHILDHOOD.}

\section{By BERNARD SCHLESINGER,}

\author{
M.A., M.D.CANTAB., M.R.C.P.LOND.,
}

Physician to the Children's Department, Royal Northern Hospital; Physician to Out-Patients, Hospital for Sick Children, Great Ormond Street Hospital.

WiTh the gradual advance of medical knowledge and the increasing help obtainable from the laboratory, the diagnosis of obscure fever is becoming somewhat simplified. Such terms as "febricula" are no longer in use, yet it served as a useful though rather unsatisfactory name for certain of the unexplained fevers of the last century. It is true that in the Great War, N when the exigencies of the moment often demanded a hasty label for a case without sufficient time for proper diagnosis, P.U.O. (pyrexia of unknown origin) was a parti- $\stackrel{?}{?}$ cularly popular classification. Such a $\frac{7}{0}$ diagnosis to-day would rightly be looked 
upon askance; yet in children there is still at times every excuse for this declaration of failure, when first confronted with a difficult case.

Children may behave in such unorthodox ways, both towards serious ailments and towards disorders that can hardly be dignified by the name of disease, that such diagnoses as " acute death" and " deep-seated mischief " are not so foolish as they sound. One has only to witness the sudden demise of an apparently thriving child with eczema, or the hyperpyrexia that can be produced in an infant through too close proximity to an insufficiently protected hot-water bottle, to realize the truth of this statement.

Children are notoriously liable to run a high temperature on the slightest provocation. The central nervous system has not yet become properly stabilized and the synapsial insulation is only in the process of completion. This has not reached such an advanced stage in some children as in others, and in those the temperature, which like all bodily functions is under the direct control of the central nervous system, is more easily influenced by extraneous causes. It is the "nervous" child who most easily becomes febrile from quite trivial effects, such as slight coryza, lumps of constipated fæces, or even unwonted excitement or an unusual indigestible addition to the diet. The mother or the attending practitioner soon recognizes this type of child and learns not to take too serious a view of any sudden unexpected pyrexia that may occur. In other children who are constitutionally subnormal in temperature, the supervention of fever is of greater significance, and it is particularly important to ascertain the cause. One should not be content to label the case " influenza," which is so often done. This is usually a cloak for ignorance, especially since true influenza is not a common ailment of childhood.

For the purposes of this lecture it is convenient to divide the subject under three headings: recurrent pyrexia, prolonged slight fever, and high temperatures.

Recurrent Pyrexia.-Cases in which a child periodically becomes suddenly ill with a high temperature and recovers in a few days without manifesting any particular group of symptoms may prove puzzling. The doctor, confronted with an illness during its acute stage, when the effects of the fever tend to obscure the main issue, is at a certain disadvantage. The consultant has an easier task. He usually sees the child later after the temperature has subsided, and can view the case from a clearer horizon, laying stress in his inquiries on certain special symptoms and on the general health of the child in the intervals between the attacks.

The origin of these obscure illnesses can usually be traced to one of two causes, either an exacerbation of a chronic pharyngitis, or an acute gastro-intestinal upset. Both may result in a few days' temperature of $103^{\circ}$ or $104^{\circ} \mathrm{F}$. and are usually ushered in by vomiting, a general feeling of malaise, peevishness, anorexia, headache, and pains in the limbs. Cough may be present when the pharynx is to blame, but the fact that children may undergo the most acute tonsillitis without complaining of sore throat rather disarms suspicion from that quarter. Bearing this in mind, no examination is ever complete in a child without searching for some sign of infection in the fauces. During the acute attack, obvious evidence of throat inflammation may be discoyered; when this is doubtful, enlargement of the tonsillar glands at the angle of the jaw will give the clue to the diagnosis. Not infrequently these never subside properly even between the attacks, and, with buried tonsils, may be the only sign of chronic infection of the throat.

When of alimentary origin, vomiting and colic are special features in the attacks, and the former may reach such severity as to be indistinguishable from the condition of acetonæmia and cyclical vomiting. The stools are pale in colour, often undigested 
or containing mucus. Inquiry will usually elucidate the statement that they are frequently unhealthy when the child is otherwise apparently ivell. A history of thread worms is sometimes obtained. Colds in such children are said always to "fly to the stomach," and should the stools in the normal course of events appear to be healthy, even a mild aperient will produce an appreciable amount of "slime" in the motions.

Thus treatment in these cases of recurrent pyrexia is usually finally focused either on the throat or on the alimentary tract. If the pharynx is implicated, a throat paint or spray and medicine containing potassium chlorate and sodium salicylate will most readily terminate the acute attack. Later, steps must be taken to eradicate any chronic focus of infection from this area; septic tonsils and adenoids may require removal, the criterion of their healthiness resting not only on their aspect alone, but extending further to the state of the corresponding cervical glands. When, on the other hand, the digestion is the seat of the trouble, a calomel purge followed by a strict diet will set matters right most quickly. These pyrexial bouts have not been loosely termed bilious attacks in vain; the vomiting and pale, fatty stools certainly point to some hepatic dysfunction. The diet should therefore be as light as possible, consisting at first only of beef tea, jellies and whey. If cornflour, Benger's food or arrowroot is given, it should be made with skimmed or diluted milk.

Much can be done to overcome the tendency to chronic intestinal indigestion in these children, and hence the periodic acute flare-up of the condition, by judicious dieting. The food should be selected so as not to leave any fermentative or irritating residue in the intestines; starchy ingredients should be cut down and the quantity of milk limited. Porridge, brown bread, raw fruit and jams containing pips, seeds and skins, and unsieved vegetables, are particularly undesirable. Rich tonics, such as cod-liver oil and virol, only aggravate the condition. The bowels need special attention and the tend-市 ency to constipation must be overcome byc the regular use of mild, non-purging, laxa- $\overrightarrow{\vec{s}}$ tives such as rhubarb, syrup of senna, or: taxol. Liquid paraffin is not well tolerated.음 Intestinal antiseptics only have a limited use $\frac{\bar{c}}{\bar{\sigma}}$ but may be worthy of trial, the best beingo small doses of hyd. cum cret., a few dropses of castor oil or salol. These measures may $\overrightarrow{0}$ effectively prevent the acute pyrexial attacks $\overrightarrow{\vec{\omega}}$ in many instances, but the condition may at $\stackrel{\circ}{\circ}$ times be obstinate and not entirely amenable을 to treatment. Fortunately, as the child gets older and the digestion becomes stronger, or the disorder as a rule disappears.

$l$ have dealt at length with these two typeso of recurrent pyrexia, for they are among the most common ailments we are called upon to treat.

The next form of obscure pyrexia we have to consider is met with just as frequently; it is apt to lead to great scares of seriogs disease, whereas often it really calls for 1 form of treatment except perhaps of the parents in certain cases.

Prolonged Slight Feier.-It should be realized at once that the normal temperature $\stackrel{\mathbb{2}}{2}$ is not such a hidebound figure that it cannot $\overrightarrow{\overrightarrow{0}}$ have slight variations in different individuals. Thus certain children constantly have a temperature below the normal accepted standard ; in them a temperature of $984^{\circ} \mathrm{F}$, may even amount to fever. On the other $\underline{3}$. hand, there are some who show a temperature in the region of $99^{\circ} \mathrm{F}$. at night without this having any significance whatever. Ito is often encountered in nervous children, their temperament being well reflected by a․ liability to habit spasms and other effects of a highly-strung nervous system. Sometimesn the discovery is first made after an acute febrile illness, following which the tempera-O ture is found not quite to settle to theo normal. Undoubtedly, in certain cases the acute illness is strictly to blame ; the thermogenic centre has been thrown off its balance, and complete regulation of the temperature 
fails to take place for some months after. In others, however, the slight supranormal temperature is found by accident through a continued routine use of the thermometer for the first time in the child's life.

The psychology of such families is truly interesting. Often it is the only child of devoted and over-anxious parents, who has been kept in bed for months following some acute yet trivial complaint. The patient receives constant overwhelming attention, and is rapidly being converted into a confirmed invalid. Morning and evening the ritual of the thermometer takes place, until one could almost imagine the child obliging with an evening temperature a few degrees above normal in order to come up to expectations. I have been shown charts in these cases with which the bedroom walls might have been adequately papered and which certainly gave a record of many weary months of the child's life.

Having excluded every possible organic cause for this slight prolonged pyrexia, it is the doctor's duty to take the plunge and order the child out of bed. The best advice to the parents would be to throw the thermometer out of the window, but this is apt to be received unfavourably by those to whom it has served as a trusted counsellor for so long. They can usually be persuaded to cease taking the temperature and to send their child to the seaside. Here the fresh air and good exercise stimulate the appetite and tone up the digestion, which has not unnaturally become sluggish by the long stay in bed. It is surprising how three or four weeks' convalescence will frequently cause a temperature to subside, which has been unsettled for months.

At times the two chronic infections already discussed, that of the nasopharynx and that of the alimentary tract, will give rise to prolonged slight fever. More than once I have found chronic nasal catarrah with persistent slight pyrexia to be due to a diphtheritic infection, not of the acute variety, but that associated with a "carrier." In such cases the child is obviously unwell and constantly peevish. Chronic otitis with otorrhœa is another source of infection in this area, which will not allow the temperature to settle.

Tubercular glands in the chest or abdomen must not be forgotten. They may be palpated in the abdomen in certain instances; in others the symptoms and history of the case will raise the suspicion. Radiological examination will reveal their presence at the hila of the lungs and, if calcified, they may also be visible in the abdomen.

Finally, a subacute rhenmatic infection, unassociated with any pain or arthritis, may lead to a low smouldering temperature for many months. In children this is not the rule, and the most active carditis can continue without any pyrexia at all.

High Pyrexia.-At the risk of being thought elementary I would like to remind you of the very high temperatures occurring in children, which in adults would give much severer symptoms and cause for alarm. The rapid variations in temperature are also peculiar to children, and it is not uncommon to see a patient with fever of $104^{\circ} \mathrm{F}$. on one evening which has subsided entirely by the next morning. With this propensity to high fever it is not surprising that the cause is often hard to find. The onset of an acute specific fever must naturally be borne in mind in every case, especially measles with its rather longer period of invasion. The rash is seldom delayed beyond the fitth day; if it has not appeared by then, all the exanthemata can practically be excluded. ${ }_{1}$, Teething in infants, which should be a physiological process, sometimes takes on pathological proportions; it can cause quite high temperatures, sometimes with disturbing meningeal symptoms. There is a decided temptation to attribute to it all the ills of infancy, and good care should be taken to exclude all other causes first. Yet, at times, nothing can be discovered beyond inflamed gums, and when the tooth finally appears, or its 
"cutting" is expedited by the use of a lancet, the temperature settles rapidly.

Acute tonsillitis may lead to prolonged high pyrexia, the origin of which may escape detection unless a thorough general examination has been made. Sufficient stress has already been laid on the importance of searching for enlarged tonsillar glands, especially when, in the absence of tonsils, the pharynx does not appear to offer sufficient explanation for the illness.

Inflammation of the middle ear is mentally associated with acute earache, but in young children the site of the pain may be difficult to locate, or it may be entirely absent. These latent cases of otitis media with high temperature and distressing illness may be very baffling and are often only finally solved by the sudden appearance of a profuse discharge. Examination of the ear would seem to offer a clear means of diagnosis ; frequently it does, and the disease can be cut short by incision of the drum. Occasionally, however, the speculum even in the most expert hands reveals no gross abnormality. It is not uncommon for an otitis to be set up by enucleation of the tonsils and adenoids through a spread of infection up the Eustachian tube. The child is brought back a week or so after the operation with an obscure and severe febrile illness, closely simulating an early meningitis, until the trouble is eventually proclaimed by the drum bursting. Similar events may result from acute tonsillitis, and in such a case the ear should always be suspected whenever the temperature is prolonged for over a week or recurs after about that interval.

Undigested food lying in the intestine may lead to irritation and absorption of the putrefying contents, causing high fever. Round worms have been recorded to lead to similar results, the temperature quickly subsiding after the worm has been passed.

One of the commonest causes of an unexplained pyrexia in girls is pyelitis. Either examination of the urine has been omitted, often owing to the difficulty of procuring it, $\frac{\$}{2}$ or the doctor has been content with macro- $c$. scopic tests for pus. In all cases of obscure $\overrightarrow{\vec{\omega}}$ fever in female children a sample of urine $\frac{+}{0}$ must be obtained, if necessary by catheteri-흐 zation. The degree of infection varies, but $\frac{\overline{\bar{p}}}{\bar{\sigma}}$ if a fair number of leucocytes are seen under $\stackrel{\mathbb{\Omega}}{\varrho}$ the microscope in a non-centrifugalized specimen the diagnosis can confidently be $\overrightarrow{0}$ made. Albumin is usually present and the $\overrightarrow{\vec{\omega}}$ reaction almost invariably acid if the urine ${ }^{\omega}$ is fresh. As a rule, Bacillus coli is the organism responsible, but I have seen typhoid fever simulate at first a simple pyelitis. B. coli pyelitis, if left untreated too or long, can become a grave menace to life, $\stackrel{\sigma}{\circ}$ and the dramatic way in which the illness? can be rapidly cured by adequate admini- 7 stration of alkalies, such as potassium citrate $\frac{\mathbb{O}}{2}$ and sodium bicarbonate, is hardly equalled in the treatment of any other children's complaint. Urinary infections are one क्ष the few ailments in childhood which are associated with rigors. During one of thes $\overline{2}$ 을 twitching of the jaw may be seen and mistaken for a fit, leading to a mistaken diagnosis of meningitis. In infants, owing to $\stackrel{\unrhd}{\complement}$ the high temperature, rapid grunting re- $\overrightarrow{\overrightarrow{0}}$ spiration may be noticed and the condition has some resemblance to pneumonia. This similarity is enhanced by the abrupt fall of temperature by crisis in some cases shortly after administration of alkalies.

"Inanition tever," first described by Holt, can be considered under the same category. 올 It occurs in young infants who are receiving insufficient food. The ingenious theory advanced is that the lack of fluid fails to flush out the kidneys properly; uric acid $\bar{N}^{\circ}$ crystals are deposited in the tubules, giving $N$ rise to an inflammatous irritation and $\mathcal{N}_{\mathrm{S}}$ consequent pyrexia.

A patch of pneumonia situated deep in the centre of the lung or at one apex is not always easily discovered, and the temperature $\stackrel{\mathcal{S}}{\rightarrow}$ may remain unexplained for several days. The disease is finally brought to light by an 
extension of the lesion in the lung giving rise to obvious physical signs, or by an abrupt termination of the illness by a crisis. When searching for pneumonia in children it is always wise to listen above both clavicles and in each axilla; by this means a small patch of consolidation is often revealed.

True infuenza was elsewhere stated to be uncommon in children, but it can occur and lead to very prolonged pyrexia. Open air is the best method of treatment when the temperature fails to subside. Cases of glandular fever in children seem to arise in groups at certain times and, although no direct relationship has been proved, they do tend to occur during influenzal epidemics. The disease was rife in the spring of this year and led to several puzzling cases of unexplained fever, particularly as in some the glandular enlargement was not very obvious, although the blood-counts were characteristic.

The clinical onset of acute leukormia may give rise to difficulty, the only manifestation sometimes being an unexplained pyrexia. Septic tonsillitis and stomatitis with red spongy gums may usher in the disease, which later becomes more apparent by the increasing pallor. Enlarged glands may not be present, but the spleen is generally easily palpable and the blood-count clinches the diagnosis.

Lymphadenoma may begin just as insidiously, and I have had a small girl under my care, who for several weeks ran a temperature of the fel Ebstein variety before the glands and enlarged spleen made their appearance.

With the present-day access to the laboratory, enteric fever is not often missed ; in fact in most cases of obscure pyrexia which one sees, the Widal reaction, if not already performed, is under contemplation. This infection, however, may be overlooked when, as so often in children, the temperature and general features of the disease do not follow the classical course. Striking lassitude and misery of the patient, together with a palpable spleen, possibly spots, and a pulse rate unduly slow in relation to the pyrexia, are useful points to mark it out.

Miliary tuberculosis has a close resemblance to typhoid fever, and is not always easy to distinguish, but here the abdomen is usually flat or retracted instead of full, and the child is more irritable. A radiogram of the chest will confirm the diagnosis, but this is sometimes hard to obtain. The importance of a thorough examination is emphasized in the case of erythema nodosum. This mild disease may cause considerable pyrexia, and if the examination stops short at the abdomen, the characteristic bluish red bumps on the legs may be overlooked. I have purposely left acute rheumatism to the last. In rheumatic fever there is usually some obvious sign of the disease. Active rheumatic carditis may be associated with a sharp bout of pyrexia, but when the fever is high, pericarditis should always be suspected. Rheumatic endocarditis is essentially not a febrile condition, and if a high, irregular pyrexia persists, subacute bacterial endocarditis may be present.

\section{POST-GRADUATE NEWS.}

From February 2 to February 13 the staff at the Chelsea Hospital for Women will undertake a course in Gynæcology. The course will occupy some mornings and/or afternoons and instruction will be by operation and by lecture. Fee $£_{55} 5 \mathrm{~s}$.

- From February 9 to February 28 a comprehensive course in Tropical Diseases will take place at the Tropical Hospital. Instruction will occupy the whole day. Fee f8 8s.

An all-day course will be given at the Queen's Hospital for Children from February 16 to February 28 . There is an abundance of material, both medical and surgical, as there are nearly 200 out-patients daily. Simple lunch and tea are kindly provided by the authorities. Fee $£ 33^{\text {s. }}$ 